[10] Aublant et Lisbonne. Bulletin mensuel de l'Office international d'Hygiène publique, 1929, 21, fasc. 10.

[11] Cantaloube. Annales de l'Institut Pasteur, 1910, p. 393 ; et La Fièvre de Malte en France, Paris, 191i, p. 7.

[12] Darbors. Société de Biologie, 21 janvier 1911, p. 102.

[13] Srmond, Thibautt et Brun. VI $I^{e}$ Congrès de l'Alliance d'Hygiène sociale, Marseille, 1910.

[14] H. Vincent. Académie de Médecine, 20 juin 1922.

[15] Vatulant. Le Lait, 1924, pp. 89-105 et pp. 200-208.

[16] Ch. Porcher. Lettre à M, le Directeur de l'Assistance et de l'Hygiène publiques, 19 décembre 1923, in Cours d'Hygiène, de L. Bernard et de R. Debré, 1, p. 369.

[17] L. F. Rosengreen. Communication de la Station centrale suédoise d'essais agricoles, $\mathrm{n}^{\circ} 215$ : Essais de Laiterie, $\mathrm{n}^{0} 20,1921$.

[18] Dubois et Sollier. Bulletin de l'Académie de Médecine, séance du 29 juillet 1930.

\title{
LES POTENTIELS DE RÉDUCTION DANS LE LAIT FRAIS, NON CUIT (1)
}

\author{
par \\ LASCAR BURUIANA \\ Ingénieur Chimiste au La boratoire de Chimie biologique de la Faculté de Médecine \\ vétérinaire de Bucarest.
}

Clark [1] a observé le premier que l'électrode de platine non platiné, c'est-à-dire à surface lisse, introduite dans le lait frais, non cuit, prend un potentiel positif de l'ordre $+0,200 \nabla$., mesuré en rapport avec l'électrode d'hydrogène.

Si l'on ajoute maintenant dans le lait de petites quantités d'une aldéhyde quelconque, le potentiel change de signe après quelque temps, se fixant près de $-0,200$ v. ClaAk suppose que ce changement est dû à l'enzyme de Schardinger, sans expliquer toutefois le mécanisme d'après lequel se produit le phénomène.

Le lait non cuit, infecté par les agents microbiens, présente encore plus nettement ce changement de potentiel, même sans l'apport de l'aldéhyde. Dans ce cas, la valeur finale du potentiel arrive aussi à $-0,200$ v. environ. La mesure des potentiels du lait se fait ordinairement dans une atmosphère d'azote pur pour éviter les influences perturbatrices de l'oxygène.

KODAMA [2] affirme qu'il n'a pas réussi à obtenir des potentiels réducteurs dans le lait frais non cuit en ajoutant de l'aldéhyde. Il attribue le phénomène réducteur observé par CLARK à l'infection bactérienne.

Dans un travail en réponse à celui de KODAma, Clark [3] con-

(1) Paru dans le Volume jubilaire du Professeur Ch. Porcher. 
firme de nouveau ses observations antérieures, attribuant l'insuccès de Kodama aux impuretés de l'azote. Il termine son travail par ces mots : "Puisque maintenant il existe un problème qui demande la répétition de ces expériences, les spéculations futures devront attendre la clarification de cette question du concours d'un troisième laboratoire. Rien n'est plus fatal au progrès de la science que le contentement de soi avec des idées préconçues; elles atténuent l'intérêt des vérifications et nuisent à la coneordance avec les faits expérimentaux. ")

Dans le but de fixer la question, nous avons répété les expériences de Clark avec les aldéhydes. Nous avons pris tous les soins nécessaires, afin de nous assurer de bons résultats, en tenant compte des critiques faites par Clark et Kodama. Les résultats obtenus confirment les observations de ClaRK (fig. I).

FIGURE I.

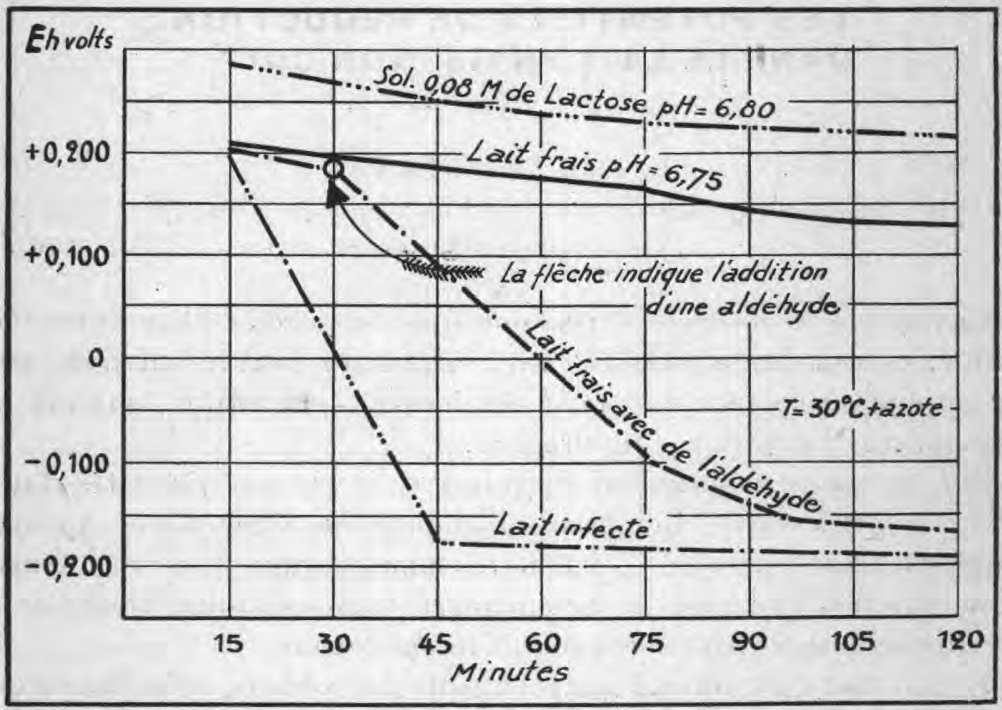

Outre les potentiels réducteurs développés par les aldéhydes ou seulement par l'infection bactérienne, nous avons observé l'apparition d'autres potentiels réducteurs tout à fait analogues, par l'échauffement du lait frais, non cuit, dans une atmosphère d'azote pur (exempt d'oxygène).

Des soins spéciaux ont été pris en ce qui concerne la purification de l'azote et la récolte du lait. Pour éviter les actions perturbatrices dues à l'infection' bactérienne, le lait était trait dans des vases parfaitement propres. Les potentiels apparus à la suite de l'échauffement étaient mesurés le plus tôt possible, tout au plus une demi-heure après. 
Les graphiques représentés dans les figures II et III montrent nettement l'influence de la température sur l'apparition des potentiels réducteurs.

\section{FIGURE II.}

Lait frais chauffé $\dot{a} 42^{\circ} \mathrm{C}$

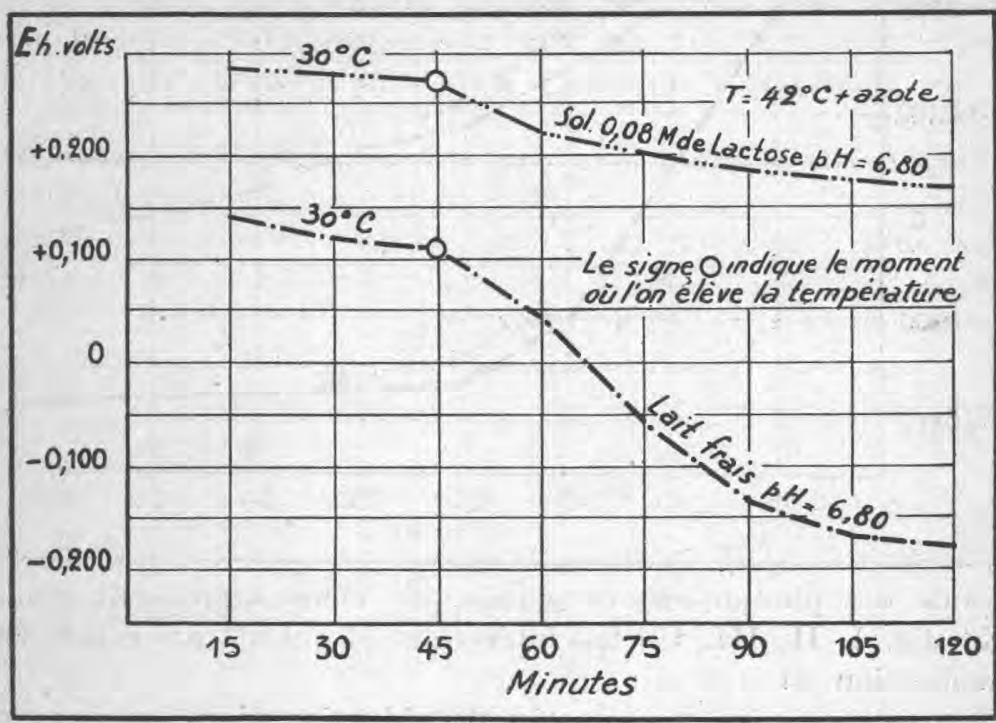

L'aspect est identique avec celui des graphiques de CLARK (fig. I) obtenus par l'addition de l'aldéhyde. La valeur finale du potentiel est, en outre, la même que eelle observée dans le cas del'intervention de l'aldéhyde et de l'infection bactérienne. Dans chaque expérience, on mesurait à la fin le $p \mathrm{H}$, électrométriquement. Les valeurs de $p$ H oseillaient dans tous les cas entre 6,65 et 6,80 .

Si l'on refroidit le lait jusqu'à $30^{\circ} \mathrm{C}$., en continuant le barbotage de l'azote, la valeur du potentiel n'est pas influencée, ou ne l'est que très peu. Les valeurs des potentiels développés sont au contraire très sensibles aux traces d'oxygène. Le potentiel du lait refroidi à $30^{\circ} \mathrm{C}$., mis en contact pendant quelques secondes avec l'atmosphère environnante, prend immédiatement la valeur positive initiale ou une valeur plus petite, mais toujours assez rapprochée.

Nous basant sur ces observations et pour expliquer l'apparition de ces potentiels, nous supposons qu'ils sont dus au lactose du lait et que c'est ce corps qui accélère, sous l'influence des enzymes déshydrogénantes et de la chaleur, l'établissement du potentiellimite. L'hypothèse paraît possible si l'on observe l'aspect des graphiques des potentiels d'une solution de lactose de la même concentration que celle du lait (solution $0,08 \mathrm{M}$. amenée à un $p \mathrm{H}=6 ; 80$ 
FIGURE III.

Lait frais chauffé à $65^{\circ} \mathrm{C}$

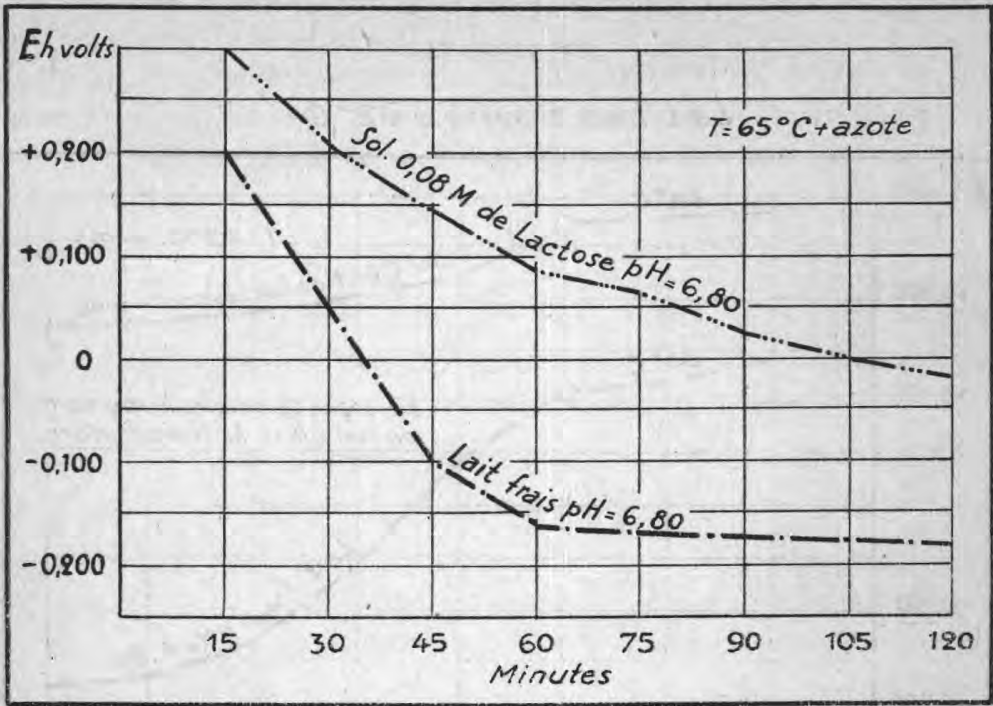

à l'aide des phosphates) et soumise au même traitement (chauffage), fig. I, II, III. Un fait intéressant et qui n'a pas encore reçu d'explication est celui-ci :

La valeur-limite du potentiel de réduction, dans les deux cas observés par ClaRK et dans le cas observé par nous, est à peu près le même et oscille autour de la valeur $-0,200 \mathrm{v}$.

\section{BIBLIOGRAPHIE.}

[1] W. M. Clank, B. Cohen et Gibbs. On oxidation-reduction. Public Health Report, Vol. 40, No. 23, 1925.

[2] Kodama. Studies on xanthine oxidase. The oxidation-reduction potential of the oxidase system. Biochem. Journ., 20, 1094, 1926.

[3] W. M. Clark, B. Cohen et M. X. Sullivan. Studies on oxidation-reduction. A note on the Schardinger reaction. In reply to Kodama. Supplement No. 66 to the Public Health Report 1927.

\section{ÉTUDE D'UNE NOUVELLE CONSTANTE POUR DÉCELER LE SALAGE DANS LE LAIT}

$$
\text { par }
$$

\section{Mlle Simone SARRAN}

Docteur en Pharmacie.

Les importants travaux de Ch. Porcher sur le lait ont établi que " lorsque le taux du lactose dans le lait vient à baisser, celui des chlorures s'élève pour assurer le maintien de l'équilibre osmotique ". 